\title{
Répertoire des genres nouveaux et des espèces nouvelles ${ }^{(1)}$
}

\section{Champignons ?}

Hepatosphaera Gambier. Incertæ sedis. Espèce type : H. molgarum Gambier. C. R. Soc. de biologie, XC, 22 février 1924, p. 441.

Hepatosphaera molgarum Gambier. Incertæ sedis. Foie. Tritons (Molge palmata, M. alpestris, M. crislala). Mulhouse (Alsace). C. R. Soc. de biologie, XC, 22 février 1924 , p. 441.

\section{Hyphomycètes}

Microsporon Ramos Horta. Conidiosporaceæ. Cuir chevelu. Enfant. Riode-Janciro. Brazil-Medico, XXXVIII, 2 février 1924, p. 59.

Trichophyton multicolor de Magalhães et Neves. Conidiosporaceæ. Cuir chevelu. Homme. Brésil. C. R. Soc. de biologie, LXXXIX, 13 octobre 1923, p. 769 .

Favotrichophyton angolense de Mello et Paes. Conidiosporacer. Cuir chevelu. Enfant. St-Paul-de-Loanda (Angola). 1er Congr. de méd. trop. de l'Afrique occidenlalc, Loanda, 1923.

M. LANGERON.

\section{Sporozoaires}

Eimeria leporis Nieschulz. Eimeridæ. Intestin grêle. Lièvre (Lepus europæus). Deutsche tierärztliche Wochenschrift, XXXI, 1923, p. 245.

Cyclospora viperae Phisalix. Eimeridx. Intestin. Vipère aspic (Vipera aspis L.). Côte-d'Or (France). Bull. Soc. pathol. exot., XVI, 14 novembre 1923, p. 637 .

Thelohania Mesnili Paillot. Microsporidie. Tissu adipeux. Chenilles de Pieris bracissæ L. Saint-Genis-Laval (Rhône). C. R. Soc. biol., XC, 29 février 1924 , p. 501.

Grahamella acodoni Carini. Sang. Acodon serrensis. Environs de São-Paulo (Brésil). Annales de Parasitologie, II, juillet 1924, p. 253.

G. LAvier.

\section{Flagellés}

Hegneria Brumpt et Lavier. Hegneridæ (Eugléniens). Espèce type : H. leplodactyli. Annales de Parasilologie, II, juillet 1924, p. 248.

Hegneria Ieptodactyli Brumpt et Lavier. Hegnerid $x$. Rectum. Têtard de Leptodactylus ocellatus (Linné). Environs de São-Paulo (Brésil). Annales de Parasitologie, II, juillet 1924, p. 248.

(1) La Direction des Annales de Parasitologie prie instamment les auteurs qui décrivent des espèces parasitaires nouvelles de vouloir bien lui adresser leurs travaux, 15, rue de l'Ecole de médecine, à Paris, afin qu'il en soit tenu compte dans le plus court délai. A défaut de tirés à part, on peut envoyer une liste des espèces nouvellement déerites, avec indications bibliographiques. 


\section{Cestodes}

Dipylidium rossicum Skriabin. Dilepinidæ. Intestin. Chien et chat. Russie. Archives de Science vétérinaire pure et appliquée (en russe). I, 1, 1923, p. 1-8.

Moniezia Chappuisi Baer. Anoplocephalidæ. Intestin grêle. Hippotragus Beckeri (Anlilopinæ). Bar el Zeruf (Nil supérieur). Revue suisse de Zoologie, XXX, 1923, p. 347.

Taeaia regis Baer. Tæniidæ. Intestin grêle. Lion (Felis leo). Bar el Zeruf (Nil supérieur). Revue suisse de zoologie, XXX, 1923, p. 347.

Taenia sp. Baer. Tæniidæ. Intestin grêle. Hyène (Crocuta maculala). Bar el Zeruf (Nil supérieur). Revue suisse de zoologie, XXX, 19:3, p. 349. (Echantillon incomplet présentant des crochets d'un type nouveau).

Prosthecocotyle Monticellii E. Linton. Telrabolhriidæ. Intestin. Globicephalus melas (Cétacés). Baie de Vineyard (Massachusset). Journ. of Parasilo$\operatorname{logy}, \mathrm{X}, 1923$, p. 51.

Anoplocephaloüdes Baer. Anoplocephalidæ. Espèce type : A. infrequens (Douthitı). Bull. Soc. neufchâteloise des Sc. nat., XLVIII, 1923, p. 10.

Echinococcus Cruzi Brumpt et Joyeux. Treniidæ. Foie et rate. Agouli (Dasyprocta aguti L.). Albuquerque Lins (Etat de São-Paulo, Brésil). Annales de Parasilologie, II, juillet 1924, p. 226.

Lüheella Baer. Lüheellidæ. Espèce type : L. pretoriensis. Annales de Parasilologie, II, juillet 1924, p. 239.

Lüheella pretoriensis Baer. Lüheellidæ. Intestin. Olocyon megalolis Desm. Environs de Prétoria. Ann. de Paras., II, juillet 1924, p. 239.

Diphyllobothrium Theileri Baer. Diphyllobothriidæ. Intestin. Zibelhailurus serval (Schreb.) et Felis caffra Desm. Environs de Prétoria. Ann. de Paras., II, juillet 1924, p. 240.

Paranoplocephala acanthocirrosa Baer. Anoplocephalidæ. Intestin. Otomys bisulcalus Cuv. Environs de Prétoria. Ann. de Paras., II, juillet 1924, p. 241.

Fuhrmannella Baer. Anoplocephalidæ. Espèce type : F. transvaalensis. Ann. de Paras., II, juillet 1924, p. 242.

Fuhrmannella transvaalensis Baer. Anoplocephalidx. Intestin. Thryonomys swinderenianus Temm. Environs de Prétoria. Ann. de Paras., II, juillet 1924 , p. 242.

Oochoristica ichneumontis Baer. Anoplocephalidæ. Intestin. Herpesles gracilis Rüpp. Environs de Prétoria. Ann. de Paras., II, juillet 1924, p. 243.

Inermicapsifer aberratus Baer. Anoplocephalidæ. Intestin. Mus moggi. Environs de Prétoria. Ann. de Paras., II, juillet 1924, p. 244.

Dipylidium Fuhrmanni Baer. Dilepinidæ. Intestin. Zibethailurus serval (Schreb.) et Felis caffra Desm. Environs de Prétoria. Ann. de Paras., II, juillet 1924, p. 245.

Hymenolepis macroscelidarum Baer. Hymenolepinidæ. Intestin. Macroscelides brachyrhynchus A. Smith. Environs de Prétoria. Ann. de Paras., II, juillet 1924, p. 246.

Taenia parva Baer. Tæniidæ. Intestin. Genelta ludia. Environs de Prétoria. Ann. de Paras., II, juillet 1924, p. 246.

Taenia hyaenae Baer. Tæniidæ. Intestin. Hyæna brunea Thunb. Environs de Prétoria. Ann. de Paras., II, juillet 1921, p. 247.

Ch. Joyeux. 


\section{Trématodes}

Sanguinicola sp. Woodland. Aporocotylida. Sang du cœur. Synodonlis schall Bl. Schm. et Auchenoglanis occidentalis Cuv. Val. (Siluridæ). Nil. Soudan égyptien. Quart. journ. micr. Soc., LXVII, (2), juillet 1923, p. 234.

Echinochasmus botauri Baer. Echinochasmidx. Intestin. Bolaurus slellaris (Ciconiiformes). Crudefin près Neufchâtel (Suisse). Bull. soc. sc. nal. Neuchâlel, XLVII, 1923, I, p. 64.

R.-Ph. Dollfus.

Opisthophallus Baer. Harmostomidx. Espèce type : O. Fuhrmanni Baer, 1923. Revue suisse de zoologie, XXX, 1923, p. 343.

Opisthophallus fuhrmanni Baer. Harmoslomida. Intestin grêle. Hippolragus Beckeri (Antilopinz). Bar el Zeruf (Nil supérieur). Revue suisse de zoologie, XXX, 1923, p. 339.

Cercaria multicellulata Miller. Monostomida. Glande digestive. Physa gyrina. Urbana (Illinois). Journ. of Parasitology, X, 1924, p. 36.

Cercaria hamata Miller. Monostomida. Planorbis lrivolvis. Urbana (Illinois). Parasilology, X, 1924, p. 37.

Cercaria elvae Miller. Furcocercaires, groupe ocellala. Limnæa stagnalis var, appressa. Lac Douglas (Michigan). Journ. of Parasilology, X, 1924, p. 38.

Cercaria Wardi Miller. Furcocercaires, groupe ocellala. Planorbis trivolvis. Urbana (Illinois). Journ. of Parasilology, X, 192t, p. 39:

Cercaria Burti Miller. Furcocercaires, groupe Donglasi, (sans indication d'hôte), Michigan. Parasilology, X, 1924, p. 40.

Cercaria tenuis Miller. Furcocercaires, groupe indicæ XXII. Planorbis trivolvis. Michigan. Journ. of Parasilology, X, 1924, p. 41.

Cercaria chrysenterica Miller. Furcocercaires, groupe emarginala. Limnæa megasoma. Michigan. Journ. of Parasilology, X, 1924, p. 42.

Cercaria intermedia W. Wunder. Zool. Anz., LVII, 1924, p. 69-83.

Ch. Joyeux.

\section{Nématodes}

Crossocephalus brevicaudatus Baylis et Daubney. Oxyuridx. Estomac. Rhinocéros de l'Inde (Rhinoceros indicus). Nepal terai, Inde. Ann. and Mag. of Nat. Hist., (9), XI, 1923, p. 333.

Tetragomphius Baylis et Daubney. Ankylostomidæ. Espèce type : T. procyonis. Ann. and Mag. of Nat. Hist., (9), XI, 1923, p. 334.

Tetragomphius procyonis Baylis et Daubney. Ankyloslomidx. Raton (Procyon sp.). Jardin zoologique de Calculta. Ann. and Mag. of Nat. Hisl., (9), XI, 1923 , p. 334 .

Setaria nudicauda Ortlepp. Filaridæe. Cervidés. Guyane hollandaise. Journ. of Helminthology, II, février 1924, p. 21.

Protospirura guianensis Ortlepp. Spiruridæ. Esophage. Monki-monki. Guyane hollandaise. Journ. of Helmintology, II, février 1924, p. 26.

Dochmoides Cameron. Strongylidz. Espèce type : D. slenocephala (Railliet, 1884). Journ. of Helmintology, II, février 1924, p. 46. 
Contracœcum haliaeti Baylis et Daubney, 1923. Helerocheilidæ. Halizlus leucogasler. Barkuda Island, Lake Chilka. Nomen novum pro C. (Ascaris) aquilla (Smith, Foxand White, 1908), Baylis, 1923. Rec. Ind. Mus., XXV, 6, décembre 1923.

$$
\text { R.-Ph. Dollfus. }
$$

\section{Acanthocéphales}

Leptorhynchoides Kostileff. Espèce type : L. plagicephalus (Westrumb, 1821). Echinorhynchus plagicephalus Westrumb, 1821. Ann. de Parasilologie, II, juillet 1924, p. 214.

M. Neveu-Lemaire.

\section{Linguatules}

Raillietiella spiralis Hett. Linguatulidæ. Poumon. Serpent. (Coeiopellis monspessulana). Palestine. Records Indian mus., XXV, juillet 1923, p. 301.

\section{Acariens}

Demodex sciurinus Hirst. Demodecidæ. Ecureuil (Sciurus vulgaris). Proc. zool. soc. London, (4), 1923, p. 971.

Laelaps Vansomereni Hirst. Gamasidæ. Rongeurs. Uganda. Annals and mag. nal. hist., XII, décembre 1923, p. 690.

Laelaps Bakeri Hirst. Gamasidæ. Rongeurs. Uganda. Annals and mag. nal. hist., XII, décembre 1923, p. 690.

Laelaps parvulus Hirst. Gamasidæ. Arvicanthis dorsalis. Smith. Rongeur. Afrique du sud. Annals and mag. nat. hist., XII, décembre 1923, p. 690.

\section{Siphonaptères}

Ctenophtalmus orphilus Jordan et Rothschild. Pulicidr. Evotomys glareolus var. helvelicus. St-Maurice, Suisse. Ectoparasiles, I, norembre 1923, p. 287.

Actenopsylla Jordan et Rothschild. Espèce type : A. suavis Jordan et Rothschild. Pulicidr. Ectoparasiles, I, novembre 1923, p. 309-319.

Actenopsylla suavis Jordan et Rothschild. Pulicidx. Nids de Plychorhamphus aleuticus. Mexique. Ectoparasites, I, novembre 1923, p. 309-319.

Hoplopsyllus exoticus Jordan et Rothschild. Pulicidr. Felis sp. Panama. Ecloparasites, I, novembre 1923, p. 309-319.

Pulex porcinus Jordan et Rothschild. Pulicidæ. Dicolyles. Texas. Ecloparasites, I, novembre 1923 , p. 309-319.

Rhadinopsylla sectilis Jordan et Rothschild. Pulicidx. Peromyscus sp. et Mus sp. Colombie britannique. Ectoparasites, I, novembre 1923, p. 309-319.

Nearctopsylla hygini var. Iaurentina. Jordan et Rothschild. Pricida. Mustela sp. Nouveau Brünswick. Ecloparasites, I, novembre 1923, p. 309-319.

F. LARrousse.

Le Gérant: F. Amirault. 